\title{
A genomic atlas of systemic interindividual epigenetic variation in humans
}

\author{
Chathura J. Gunasekara', C. Anthony Scott ${ }^{1}$, Eleonora Laritsky¹, Maria S. Baker ${ }^{1}$, Harry MacKay ${ }^{1}$, Jack D. Duryea ${ }^{1}$, \\ Noah J. Kessler ${ }^{3,4}$, Garrett Hellenthal ${ }^{2}$, Alexis C. Wood', Kelly R. Hodges ${ }^{5}$, Manisha Gandhi', Amy B. Hair ${ }^{6}$, \\ Matt J. Silver ${ }^{3}$, Sophie E. Moore ${ }^{3,4}$, Andrew M. Prentice ${ }^{3}$, Yumei Li ${ }^{7,8}$, Rui Chen ${ }^{7,8}$, Cristian Coarfa ${ }^{*^{*}}$ and \\ Robert A. Waterland ${ }^{1,8^{*}}$ (D)
}

\begin{abstract}
Background: DNA methylation is thought to be an important determinant of human phenotypic variation, but its inherent cell type specificity has impeded progress on this question. At exceptional genomic regions, interindividual variation in DNA methylation occurs systemically. Like genetic variants, systemic interindividual epigenetic variants are stable, can influence phenotype, and can be assessed in any easily biopsiable DNA sample. We describe an unbiased screen for human genomic regions at which interindividual variation in DNA methylation is not tissue-specific.

Results: For each of 10 donors from the NIH Genotype-Tissue Expression (GTEx) program, CpG methylation is measured by deep whole-genome bisulfite sequencing of genomic DNA from tissues representing the three germ layer lineages: thyroid (endoderm), heart (mesoderm), and brain (ectoderm). We develop a computational algorithm to identify genomic regions at which interindividual variation in DNA methylation is consistent across all three lineages. This approach identifies 9926 correlated regions of systemic interindividual variation (CoRSIVs). These regions, comprising just 0.1\% of the human genome, are inter-correlated over long genomic distances, associated with transposable elements and subtelomeric regions, conserved across diverse human ethnic groups, sensitive to periconceptional environment, and associated with genes implicated in a broad range of human disorders and phenotypes. CoRSIV methylation in one tissue can predict expression of associated genes in other tissues.
\end{abstract}

Conclusions: In addition to charting a previously unexplored molecular level of human individuality, this atlas of human CoRSIVs provides a resource for future population-based investigations into how interindividual epigenetic variation modulates risk of disease.

\section{Background}

Methylation of cytosines in CpG dinucleotides is an epigenetic mechanism with essential roles in mammalian development $[1,2]$. To explore its functions in cellular differentiation, unbiased analysis of CpG methylation by whole genome bisulfite sequencing (WGBS) has been used to characterize epigenetic differences among different human tissues and cell types [3, 4]. Meanwhile, human interindividual variation in DNA methylation that is not cell type-specific has attracted relatively little

\footnotetext{
* Correspondence: waterland@bcm.edu; coarfa@bcm.edu

'USDA/ARS Children's Nutrition Research Center, Department of Pediatrics,

Baylor College of Medicine, Houston, TX, USA

${ }^{9}$ Department of Molecular and Cellular Biology, Baylor College of Medicine, Houston, TX, USA

Full list of author information is available at the end of the article
}

attention. Systemic interindividual epigenetic variation is important, however, because like genetic variation it is a potential determinant of phenotype and can be assessed in any easily biopsiable DNA sample. Hence, though not the only type of epigenetic variation that might contribute to disease, systemic interindividual variants are highly advantageous for population studies. Moreover, because systemic epigenetic variants originate in the early embryo [5], their establishment can be influenced by periconceptional environment $[6,7]$.

Previous studies identified systemic interindividual variation (SIV) in DNA methylation by genome-scale DNA methylation profiling in multiple tissues from multiple individuals $[5,6,8]$, but were limited by the profiling technique and/or the number of tissues and individuals studied. Here, we performed unbiased genomewide DNA 
methylation analysis in post-mortem thyroid, heart, and brain (representing all three germ layer lineages) from each of 10 donors in the NIH Genotype-Tissue Expression (GTEx) program [9] (Fig. 1a).

\section{Results}

\section{Screen for systemic interindividual variation in DNA methylation}

We performed deep WGBS on all 30 samples, generating $\sim 1.2 \mathrm{~B} 150$-bp paired-end reads $(\sim 122$ Gb uniquely mapped sequence per sample), yielding average genomewide read depth of $\sim 40 \times$ (Additional file 1: Table S1). Genetic identity of the three libraries representing each individual was verified by calling single nucleotide variant (SNV) genotypes from the WGBS data (Additional file 4: Figure S1). We analyzed CpG methylation at 100-bp resolution, focusing on all 100-bp human autosomal bins containing at least one $\mathrm{CpG}$ site and yielding adequate read depth (see Additional file 3 for methods). Considering all $(\sim 13 \mathrm{M})$ such informative bins, genomic CpG methylation in the 30 samples clustered by tissue (Fig. 1b). Since combined effects across multiple neighboring CpGs are both more stable and more likely to affect gene expression [10], our goal was to identify regions of SIV. To maximize genomic coverage, we applied a two-stage approach. First, all reads from each individual were combined to calculate individual-level average methylation for each bin, and individually correlated regions of methylation (bin-bin $\left.R \geq 0.71\left(R^{2} \geq 0.5\right)\right)$ were built in a step-wise fashion $[11,12]$ (see "Methods" and Additional file 3: Supplementary Methods). Then, for each such region, inter-tissue correlation (ITC) of average methylation was assessed across all tissue-type pairs. By this approach, 90\% of all human autosomal bins were informative. (Sex chromosomes were not evaluated due to the limited sample size.) Regions yielding a minimum ITC $\geq 0.71$ were identified as "correlated regions of SIV" (CoRSIVs) (Fig. 1c, d).

Permutation testing showed that only 39,424 (9\%) of these 446,665 regions satisfy the minimum ITC criterion by chance; hence, the vast majority of the 39,424 CoRSIVs (Additional file 2: Table S2) are statistically significant. Many, however, include only a few CpGs, and some showed relatively minor interindividual variation (Fig. 1e). To target the most robust epigenetic variants likely to have the greatest biological relevance, we focused all subsequent analyses (and the remainder of this report) on the 9926 CoRSIVs containing at least 5 CpGs (Additional file 4: Figure S2a, b) and exhibiting an inter-individual methylation range of at least $20 \%[6,13]$ (Fig. 1e; annotated list in Additional file 1: Table S3). Importantly, analysis of our permutation results showed that each of these is statistically significant $(P<0.05)$ (Additional file 4: Figure S2c). Unlike at genome-wide bins (Fig. 1b), methylation at these 9926 CoRSIVs clustered by individual (Fig. 1f). Notably, although our cutoff for minimum ITC was 0.71, most of the 9926 CoRSIVs exhibit minimum inter-tissue $R>0.85$ (Additional file 4: Figure S2d). Analysis of DNA methylation by bisulfite pyrosequencing in an independent set of Vietnamese cadaver tissues (liver, kidney, and brain from 17 individuals) validated systemic interindividual variation at 9 of 11 loci examined (Additional file 4: Figure S3, Additional file 1: Table S4). The two that did not validate showed low interindividual variation in the Vietnamese, which might arise from chance similarities in the small sample size studied. Previous studies have estimated that $15-20 \%$ of CpG sites in the human genome show tissue-specific interindividual variation $[4,14]$. CoRSIVs are exceedingly rare by comparison, comprising only $\sim 0.1 \%$ of the human genome.

Since thyroid, heart, and brain do contain some cell types in common (white blood cells, for example) one might suppose that CoRSIV signals could arise from interindividual variation in the proportional representation of these contaminating cell types. Given the very small proportion of blood cells in the three tissues, it is difficult to imagine that they could explain inter-tissue $R^{2} \geq 0.50$. Nonetheless, we devised several tests of this alternative explanation. First, analyzing publicly available WGBS data on monocytes isolated from six individuals, we show interindividual variation at CoRSIV but not control regions (Additional file 4: Figure S4), demonstrating that the interindividual variation is evident even in a single highly purified cell type. Second, using samples collected as part of a study of infant twins, we performed bisulfite pyrosequencing at several CoRSIVs in 20-30 individuals and found that interindividual variation in fingernail DNA is correlated with that in peripheral blood (Additional file 4: Figure S5). Since fingernails are composed of keratinized nail matrix cells (and contain no leukocytes) these correlations clearly are not the result of blood contamination. Lastly, we used data from the Blueprint epigenome project [15] to assess the overlap of CoRSIVs with differentially methylated regions (DMRs) distinguishing two major leukocyte subtypes: B cells and neutrophils. If CoRSIV signals result from individual variation in the proportionality of contaminating leukocytes, CoRSIVs should overlap extensively with these DMRs. However, only 37 such overlaps were found (i.e., $<1 \%$ of the 9926 CoRSIVs) (see Additional file 3 for methods). The single explanation consistent with all of these observations is that CoRSIVs are bona fide regions of systemic interindividual variation in DNA methylation. Indeed, CoRSIVs are highly enriched for CpG sites previously shown to be highly correlated across four brain regions and blood [16] (odds ratio 16.5, $P=1.2 \times 10^{-81}$ ) (see Additional file 3 for methods). 
a
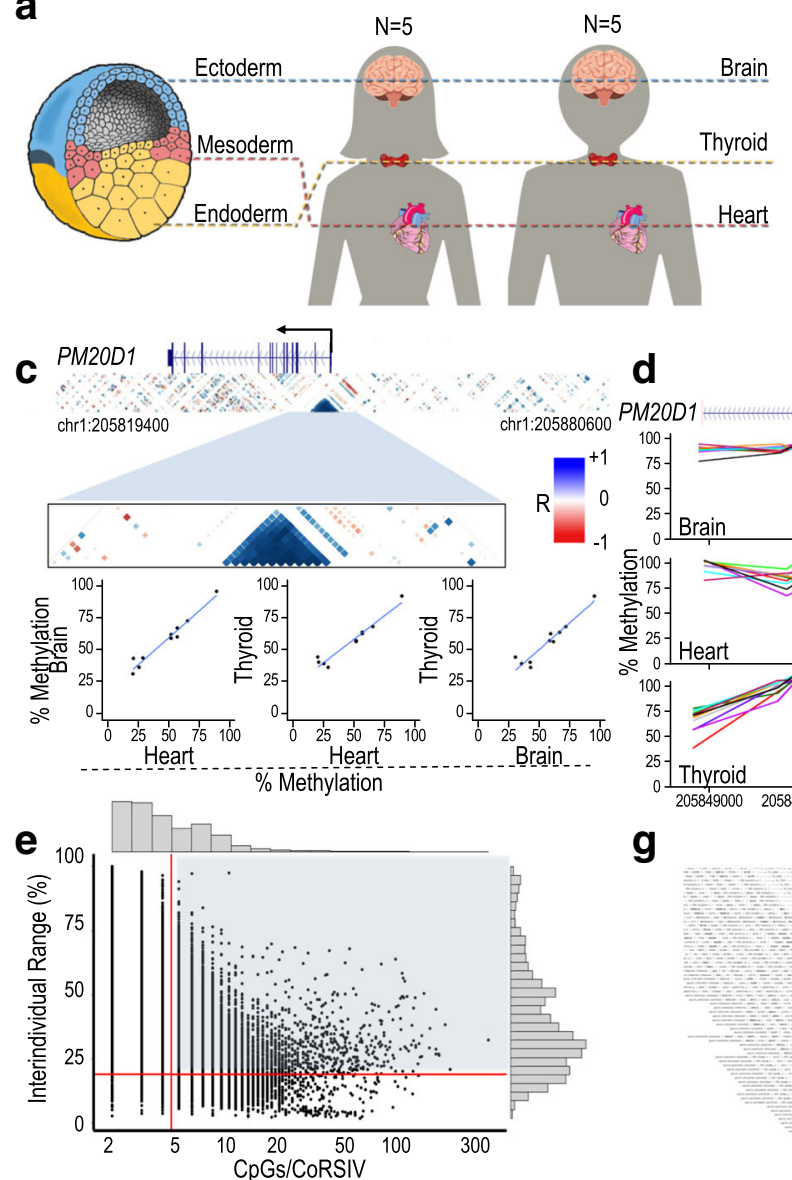

f

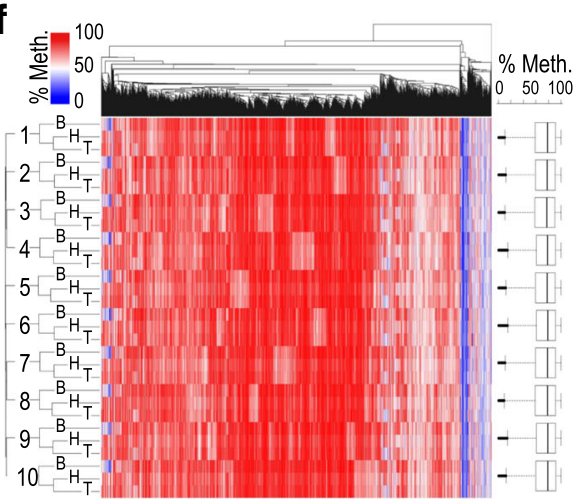

b

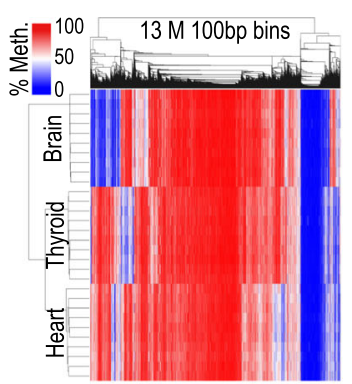

d



g

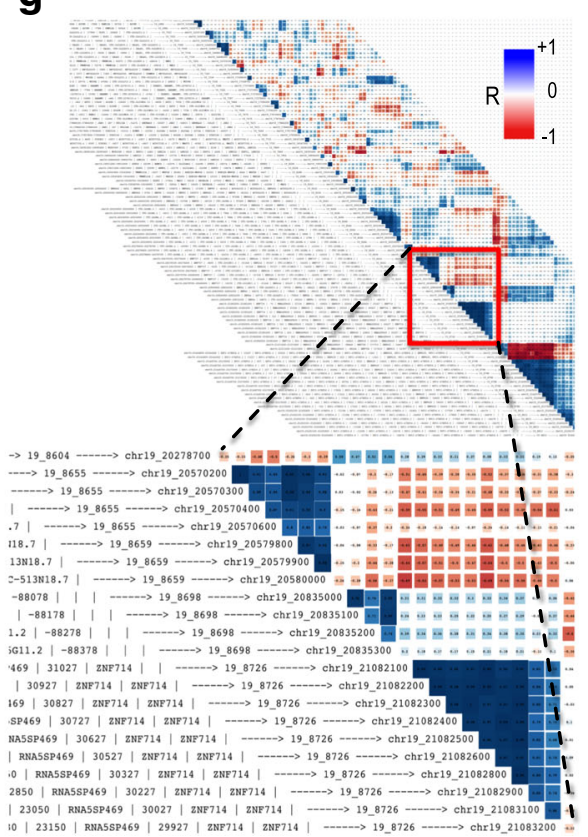

Fig. 1 Strategy for identifying correlated regions of systemic interindividual variation (CoRSIVs). a The tissues analyzed represent the three germ layer lineages; 10 Caucasian GTEx donors were studied, yielding 30 methylomes. b Initial unsupervised clustering of whole-genome bisulfite sequencing data; considering all informative bins, they cluster by tissue. $\mathbf{c}$ Example of a CoRSIV identified at the PM20D1 promoter. The blue triangle shows a region of correlated methylation comprising thirteen 100-bp bins; the three scatter plots illustrate its high inter-tissue correlation. $\mathbf{d}$ Plots of individual methylation at the PM20D1 CoRSIV illustrate systemic interindividual variation. Genotype data at rs708727 (bottom panel) indicate strong mQTL at the locus. e Scatter plot of interindividual methylation range vs. number of CpGs per CoRSIV, for all 39,424 CoRSIVs initially identified. Subsequent analyses focus on the 9926 CoRSIVs with $\geq 5$ CpGs/CoRSIV and IIR $\geq 20$ (shaded area). f Unlike genome-wide bins, the 9926 CoRSIV bins cluster by individual (B, H, T-brain, heart, thyroid). Box plots on right show that the 10 donors show no individual differences in average methylation across all the CoRSIVs. $\mathbf{g}$ An illustrative region from the CoRSIV plot of chr19. Inset shows example of annotation of a CoRSIV (chr19_8726) at ZNF714 


\section{Characteristics of CoRSIVs}

Systemic individual differences in global DNA methylation could be attributed to genetic variants in, for example, genes regulating one-carbon metabolism or DNA methylation enzymes [17] or to technical variation in, for example, the time between death and tissue collection (post-mortem interval). In selecting donors we attempted to minimize potential sources of variation including age, body mass index, and post-mortem interval (Additional file 1: Table S5). Average CoRSIV methylation did not differ among the 10 donors studied (Fig. If and Additional file 4: Figure S6a), arguing against global regulation of or systematic influences on methylation at these regions. To visualize CoRSIVs throughout the genome, we generated an atlas of annotated CoRSIV maps for each human autosome (https://corsiv.shinyapps.io/CoRSIV_Plotter/) (example region shown in Fig. 1g). A striking feature of these maps is the extensive long-range correlation (and anti-correlation) among CoRSIVs. Long-range interindividual correlation in DNA methylation was previously reported in population studies of peripheral blood methylation using a commercial methylation array $[12,18]$. For example, two regions separated by $\sim 20 \mathrm{~kb}$ and overlapping the $5^{\prime}$ and $3^{\prime}$ ends of SPATC1L, previously identified as regions of anticorrelated methylation in peripheral blood [12], are in fact anticorrelated CoRSIVs (Additional file 4: Figure S7a). Since most haplotype blocks in Caucasians are $<50 \mathrm{~kb}$ in length [19], we were surprised to observe many examples of positively intercorrelated CoRSIV pairs spanning much larger genomic distances, which we refer to as "superCoRSIVs" (Additional file 4: Figure S2f). Topologically associated domains (TADs) are broad genomic regions (median size $\sim 1 \mathrm{Mb}$ ) with a high probability of physical association and are largely invariant across different cell types [20]. We therefore tested whether superCoRSIVs tend to occur within TADs; indeed, across 10 human tissues, the proportion of superCoRSIVs wholly within TADs was consistently elevated relative to that of a set of matched control regions (paired $t$-test $P=10^{-5}$, Additional file 4: Figure S2f). Since TAD boundaries are associated with CTCF sites [20], our observation that CTCF binding sites are enriched within SuperCoRSIVs $\left(\chi^{2}\right.$ test $P=0.003$, Additional file 4: Figure S2 g) additionally suggests a mechanistic link between SuperCoRSIVs and TADs.

Whereas most of the 9926 CoRSIVs are only 200300-bp long and include 5-10 CpGs, the largest span several $\mathrm{kb}$ and involve hundreds of CpGs (Fig. 2a). Rather than being randomly distributed throughout the genome, CoRSIVs tend to occur in clusters (Kolmogorov-Smirnov test vs. uniform distribution: $P<10^{-100}$ ). The two biggest peaks of CoRSIV density are observed at the major histocompatibility (MHC) locus on chromosome
6 and the pericentromeric region on the long arm of chromosome 20 (Fig. 2b); illustrative profiles of individual methylation at one gene from each region are shown in Fig. 2c. SIV in DNA methylation was previously reported in the MHC locus [21]. We are not aware of previous publications, however, highlighting the exceptional epigenetic behavior of the chromosome 20 region. Interestingly, whereas the 92 CoRSIVs spanning the MHC region are largely independent of one another (Additional file 4: Figure S8a,b), the 56 CoRSIVs comprising the chromosome 20 region are highly intercorrelated (Additional file 4: Figure S8c,d), indicating that individual methylation status is correlated across this entire $\sim 2.3-\mathrm{Mb}$ region. As suggested by the circos plot (Fig. $2 \mathrm{~b}$ ) and the higher-resolution plots in Additional file 4: Figure S9, CoRSIVs are $>2$-fold enriched in subtelomeric regions $\left(\chi^{2}\right.$ test $P<10^{-300}$, Fig. 2d, Additional file 1: Table S6). Given that subtelomeric regions are highly variable, one might suppose that the CoRSIV enrichments in these regions might be due to poor mapping rates leading to artifacts. However, unique mapping rates in the chromosome 20 pericentromeric region were generally above, and those in subtelomeric regions only slightly below, the genomewide average (Additional file 4: Figure S10). Further, only 14 of the 9926 CoRSIVs overlapped an ENCODE blacklist region [22] (genomic regions known to yield artifacts in functional genomics studies) (see Additional file 1: Table S19).

To explore sequence features associated with CoRSIVs, we generated two reference sets of genomic regions: a randomly selected set of genomic regions matched to the CoRSIVs on chromosome, size, and CpG content ("controls") (Additional file 4: Figure S11a, Additional file 1: Table S7), and a set of similarly matched loci drawn from regions of tissue-specific differential methylation (tDMRs) (Additional file 4: Figure S11b,c, Additional file 1: Table S8). Compared to both reference sets, CoRSIVs are enriched for transposable elements and depleted for $\mathrm{CpG}$ islands (CGI) and transcription factor binding sites $\left(\chi^{2}\right.$ test $P<10^{-8}$ for all comparisons; Fig. 2e, Additional file 4: Figure S2 h, Additional file 1: Table S9). Relative to either controls or tDMRs, CoRSIVs are under-represented within and near genes and enriched in intergenic regions $\left(\chi^{2}\right.$ test $P<10^{-16}$; Fig. 2f, Additional file 1: Table S10). Analysis of ChromHMM features across 111 reference human epigenomes (derived from the NIH Epigenome Roadmap project) [23] shows that, relative to control regions, CoRSIVs are enriched in quiescent regions and those associated with repressive polycomb marks and depleted in heterochromatic regions, active promoters, and enhancers (Fig. 2g); the strongest depletions were found for promoter and enhancer regions characterized as "bivalent" (i.e., poised between active and inactive states) in embryonic stem cells [24]. Similar depletions/enrichments are observed when CoRSIVs 

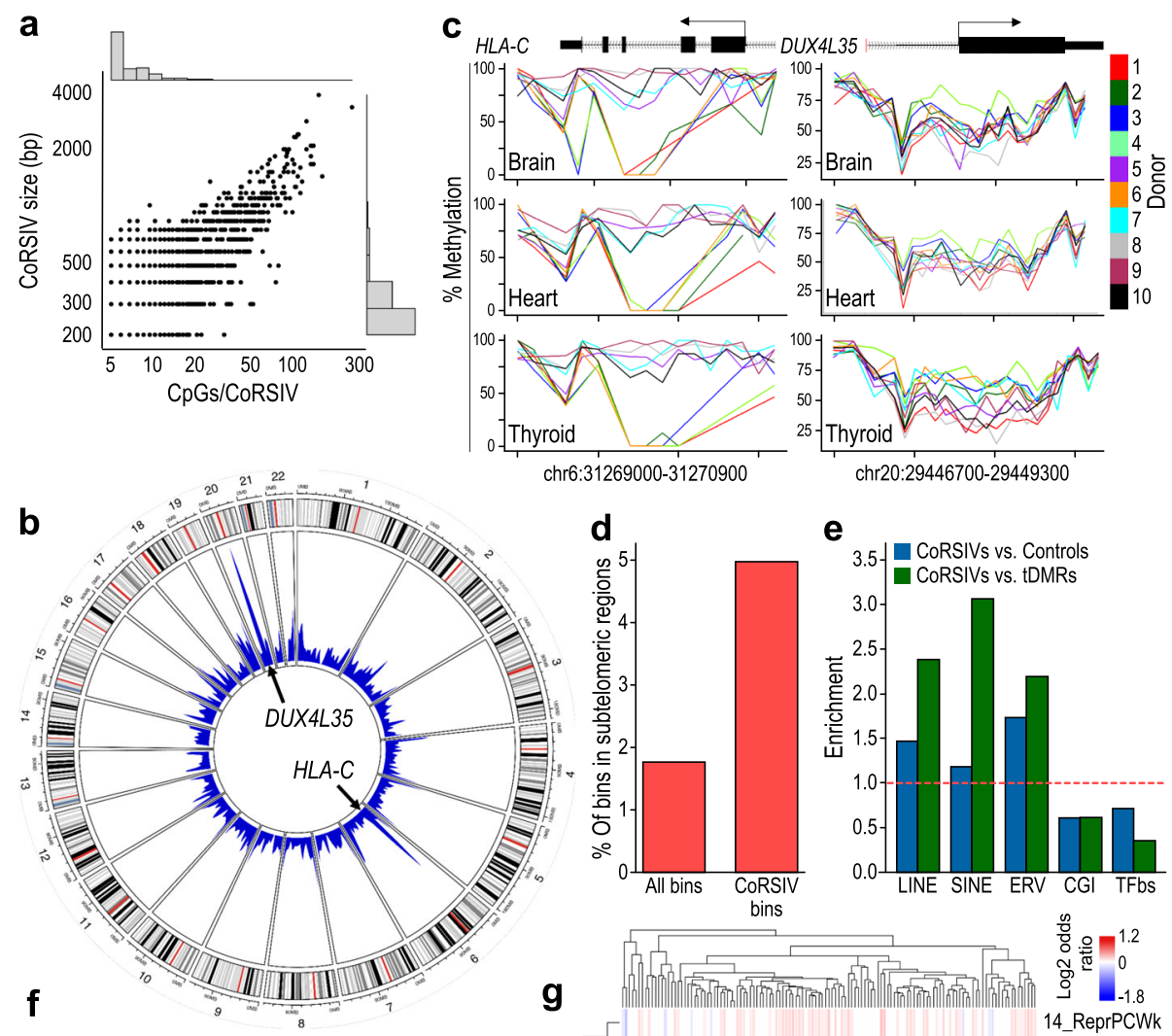

e


Fig. 2 Distribution and characteristics of 9926 human CoRSIVs. a Scatter plot of CoRSIV size vs. CpGs per CoRSIV. b Circos plot of human autosomes indicates regions of high CoRSIV density. $\mathbf{c}$ Individual methylation levels across examples of CoRSIVs from each of the two most high-density regions: (left) HLA-C (chromosome 6) and (right) DUX4L35 (chromosome 20). d Compared to all informative bins, CoRSIVs are more than twofold enriched in sub-telomeric regions $\left(X^{2}\right.$ test $\left.P<10^{-300}\right)$. e Compared to control regions or tissue-specific differentially methylated regions (tDMRs), CoRSIVs are enriched for repetitive elements and depleted for CpG islands (CGI) and transcription binding sites (TFbs) $\left(X^{2}\right.$ test $P<10^{-8}$ for all comparisons). $\mathbf{f}$ Relative to control and tDMR regions, CoRSIVs are under-represented at transcription start sites (TSS), within gene bodies, and at transcription end sites (TES) ( $X^{2}$ test $P<10^{-16}$ for all comparisons). $\mathbf{g}$ ChromHMM epigenomic features significantly enriched or depleted in CoRSIVs

are compared to tDMRs (Additional file 4: Figure S2e, Additional file 1: Tables S11, S12). Analysis of data on genomic evolutionary rate profiling scores [25] showed that, relative to both control and tDMR regions, CoRSIVs tend not to occur in highly conserved genomic regions (CoRSIVs vs. controls odds ratio $=0.4, P=8.2 \times 10^{-118}$; CoRSIVs vs. tDMRs odds ratio $=0.2, P<1.0 \times 10^{-200}$ ) (Additional file 4: Figure S2i).

\section{Influence of genetic variation on CoRSIVs}

We took several complementary approaches to evaluate the extent to which DNA methylation at CoRSIVs is genetically determined. Associations between genetic variation and DNA methylation can be assessed relative to single genetic variants (methylation quantitative trait loci-mQTL) [26, 27] or at the haplotype level (haplotype-dependent allelespecific methylation) [28]. As previously reported [29], the 
PM20D1 promoter region illustrated in Fig. 1d exhibited strong mQTL. With methylation data on only 10 individuals, however, we were underpowered to analyze mQTL at all 9926 CoRSIVs, so performed focused analyses on several regions of high CoRSIV density (Fig. 2b). We used donor-specific SNV data from GTEx $[9,30]$ to test for associations between individual-average CoRSIV methylation and SNV genotype. Since the strongest mQTL effects occur over fairly short distances [28], we assessed associations with common variants within $10 \mathrm{~kb}$ of each CoRSIV and, to minimize false positives, considered only SNVs for which all three genotypes were represented. Of 96 informative CoRSIVs in the high-density region overlapping the MHC locus on chromosome 6 (Fig. 2b, Additional file 4: Figure S8a), 74 showed significant mQTL (Fig. 3a, left), corroborating a previous report that the MHC locus is an mQTL hotspot [12]. Conversely, in the high-density CoRSIV region near the chromosome 20 centromere (Fig. 2b, Additional file 4: Figure S8c), none of 10 informative CoRSIVs showed mQTL (Fig. 3a, right). Next, we considered all pairwise comparisons among the 10 donors to test associations between interindividual differences in methylation and average SNV genotype in the 10-kb regions flanking each CoRSIV. This analysis (Fig. 3b) indicated that $~ 60 \%$ of interindividual variation in CoRSIV methylation is explained by genetic variation in cis. We also exploited several published mQTL data sets based on the Illumina HM450 array $[26,27,31-33]$. Of the $\sim 485,000 \mathrm{CpG}$-specific probes included on the HM450 array, just 1659 overlap 819 CoRSIVs (Additional file 1: Table S13). Across eight populations studied by five different groups, approximately half of these CoRSIVs consistently showed mQTL, while $\sim 40 \%$ showed no evidence of mQTL (Fig. 3c, Additional file 1: Table S14). The methylation distribution in each of the 10 GTEx donors is substantially different for mQTL-negative vs. mQTL-positive CpGs (Additional file 4: Figure S6b), providing independent corroboration of the two classes evident in the HM450 mQTL analysis.

We next used the DECIPHER database [34] to test whether CoRSIVs might be explained by copy number variations (CNVs). Unsurprisingly (given their association with subtelomeric regions), CoRSIVs were significantly enriched for overlaps with $\mathrm{CNV}$ regions compared to control (odds ratio $=1.8, P=1.7 \times 10^{-94}$ ) and tDMR regions (odds ratio $=2.7, P=8.1 \times 10^{-100}$ ). To determine if SIV among the 10 GTEx donors is associated with deletions or duplications, we calculated Spearman correlations between average WGBS read depth and average CoRSIV methylation across the 10 individuals. The $P$ value distribution (Fig. $3 \mathrm{~d}$ ) shows that, for the vast majority of CoRSIVs, individual-average methylation is not significantly associated with read depth, indicating that CNV generally does not explain SIV at CoRSIVs.
Previous population-based studies have examined haplotype effects on regional methylation by comparing the decay rate of correlation in methylation with that of genetic linkage [11, 12]. We likewise analyzed methylation data at CoRSIVs in the context of linkage disequilibrium (LD) data on Caucasian individuals (CEU) in the 1000 genomes project [35]. Relative to control regions, CoRSIVs were associated with regions of higher LD, and methylation in CoRSIVs was more highly correlated with that in flanking regions (Fig. 3e). Whereas previous studies on methylation variants in Arabidopsis [11] and humans [12] found that methylation correlation decays very rapidly (within $1-2 \mathrm{~kb}$ ), methylation decay at CoRSIVs is much longer, not returning to baseline until $\sim 20 \mathrm{~kb}$ (Fig. 3e). To examine regional associations between genetic and methylation variation, we therefore assessed the linear correlation between methylation decay and LD decay within $20 \mathrm{~kb}$ upstream and downstream of each CoRSIV. The resulting $P$ value distribution (Fig. 3f, Additional file 1: Table S15) is highly enriched for significant values, providing evidence of genetic influence on methylation. Examples drawn from opposite ends of the $P$ value distribution illustrate that this approach distinguishes CoRSIVs located entirely within haplotype blocks-and putatively under genetic control-(Fig. 3g) from those in which strong regional correlation in methylation occurs in the absence of underlying haplotype structure (Fig. 3h), indicating "pure" epigenetic variation [11].

Together, these analyses show that methylation at many CoRSIVs is influenced by genetic variation in cis but also suggest that, at some of these loci, systemic interindividual epigenetic variation may be independent of genetic variation. With the data currently available, we cannot yet make definitive conclusions about the absence of genetic effects. Characterization of all possible genetic influences on CoRSIV methylation (including trans effects, which were not evaluated here) will require methylation analyses targeted to these regions in a large number of genotyped individuals. Systemic interindividual epigenetic variation that is largely independent of genetic variation is consistent with metastable epialleles [36]. In mouse and human studies, epigenetically metastable loci are characterized by stochastic interindividual variation in epigenotype that is established in the early embryo [5] and influenced by periconceptional environment $[7,8,37,38]$.

\section{Influence of periconceptional environment on CoRSIVs}

To assess potential epigenetic metastability at these loci, we examined data from a natural experiment in a well-characterized population of subsistence farmers in rural Gambia in which annual climatic variation causes dramatic seasonal variation in maternal nutritional 


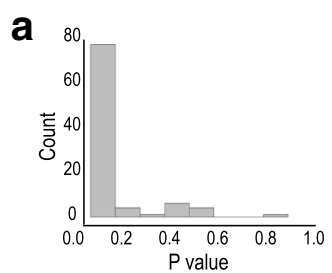

b

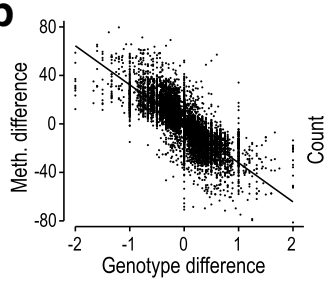

d



g

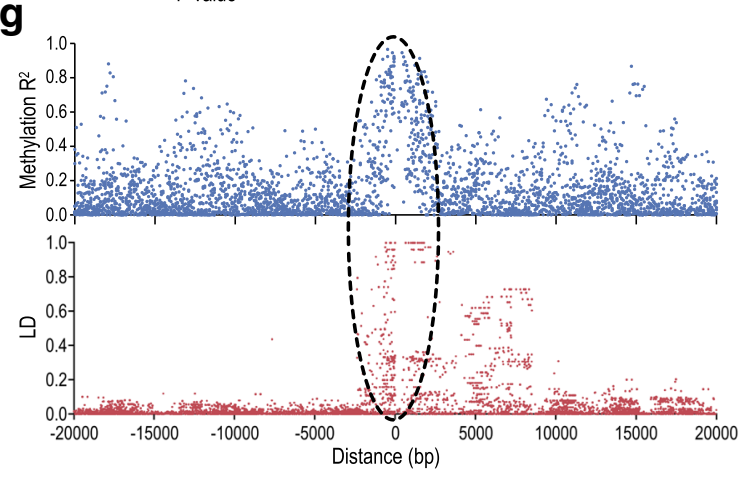

h

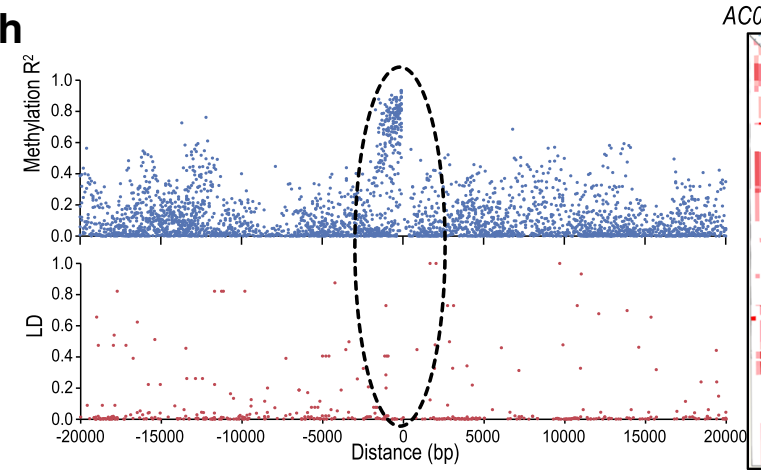

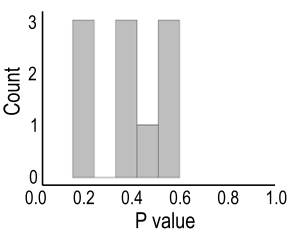



e



f
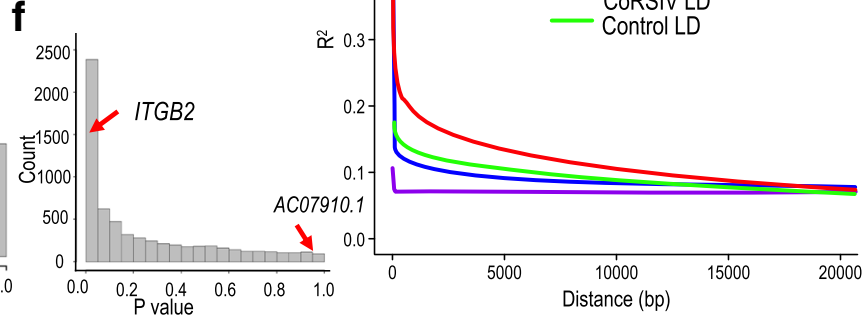

ITGB2 region ( chr21: 44910446-44928061)

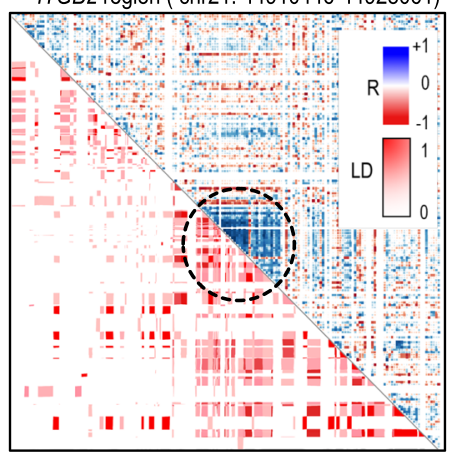

AC079610.1 region (chr2:212825049-212840256)



Fig. 3 (See legend on next page.) 
(See figure on previous page.)

Fig. 3 Evaluating associations between CoRSIV DNA methylation and neighboring genetic variation. a CoRSIV-specific $P$ value distributions from $\mathrm{mQTL}$ analyses at the two most CoRSIV-dense genomic regions. At the MHC locus on chromosome 6, most CoRSIVs show mQTL; none of 10 informative CoRSIVs in the pericentromeric region on chromosome 20 show mQTL. b Summary of pair-wise analyses. Scatter plot shows correlation of interindividual differences in CoRSIV methylation vs. interindividual differences in average CoRSIV-associated SNV genotype for one pair of GTEx donors (RVPV and X261). Distribution of all such pairwise comparisons (right); average $R^{2}=0.61$. c Heat map of published $\mathrm{mQTL}$ effects at 819 CoRSIVs overlapping probes on the HM450 array. Approximately 40\% (white) show no evidence of mQTL. Dotted line shows location of probes within the PM20D1 CoRSIV. d Histogram of $P$ values for the spearman correlation between methylation and read coverage within each CoRSIV. Two insets above the histogram show two scatter plots drawn from opposite ends of the histogram. The top left inset shows a strong association $(P<0.05)$ between average methylation in chr3_2398 CoRSIV and individual-average read depth in this region. The top right inset shows weaker association $(P>0.85)$ between average methylation in chr6_24299 CoRSIV and individual-average read depth in this region. e Decay curves of correlation in methylation and LD for CoRSIVs and control regions. f Histogram of CoRSIV-specific $P$ values for the Spearman correlation analyses of methylation decay vs. LD decay. Locations of two CoRSIVs with (ITGB2) and without a significant association (AC07910.1) are indicated. $\mathbf{g}$ The CoRSIV at ITGB2 yielded a significant association $(P<0.05)$ between methylation decay and LD decay, consistent with a haplotype-effect on methylation. The scatter plot on the left (top, blue) shows all methylation $R^{2}$ for pairs of bins in which one bin is within the CoRSIV and other bin is within $\pm 20 \mathrm{~kb}$. The scatter plot on the left (bottom, red) shows LD for all pairs of SNVs. For each pair one SNV is within the CoRSIV and the other is within 20kb. On the right, the upper diagonal shows the CoRSIV (blue) in this region, and the lower diagonal shows the LD pattern in this region (1000 Genomes CEU). $\mathbf{h}$ In contrast, the CoRSIV at AC07910.1 showed no association between methylation decay and LD decay $(P>0.9)$, suggesting pure epigenetic variation

status and energy balance. Previous studies in this population show that, at candidate and bona fide metastable epialleles, children conceived during the peak of the rainy season show persistent and systemic elevations in DNA methylation compared to children conceived during the dry season, and these correlate with the mothers' methyl donor metabolomes assessed in early gestation [37]. Here, rather than focus on pre-selected epochs, we studied 233 children conceived throughout the calendar year [13] and performed Fourier regression analysis to determine seasonal effects agnostically. DNA methylation in peripheral blood was measured by Illumina HM450 arrays, so this analysis is limited to CoRSIVs and control regions that are informative on this platform. Significant seasonal variation was highly enriched at CoRSIVs (Fisher's exact test $P=1.7 \times 10^{-6}$ ) but not at controls or tDMRs (Fisher's exact test $P=1.0$ and 0.85 , respectively, Additional file 4: Figure S12a,b). Moreover, this unsupervised analysis independently validated seasonal effects corresponding to peaks of the rainy (July-September) and dry (January-April) seasons (Fig. 4a) consistent with previous studies of human metastable epialleles $[6,8,13,37]$. It should be noted that in these outbred human populations we have yet to rule out potential confounding by genetic variants that influence both season-specific fecundity and systemic methylation. This caveat notwithstanding, these data provide independent evidence that a significant proportion of CoRSIVs exhibit epigenetic metastability, which can be influenced by maternal nutrient status during early pregnancy.

\section{Associations of CoRSIVs with gene expression and human disease}

CoRSIVs show great promise for epigenetic epidemiology [39] because, at these loci, DNA methylation measurements in any easily biopsied tissue should provide information about epigenetic regulation throughout the body. To test this, we analyzed data from a previous large population study of adult women in which DNA methylation in adipose tissue was measured by the HM450 array and gene expression was assessed in adipose tissue, skin, and lymphoblastoid cell lines [26]. Unlike at control regions and tDMRs (Additional file 4: Figure S13b), among gene-associated CoRSIVs that showed an association between methylation and expression in adipose tissue, most also showed an association between methylation in adipose tissue and gene expression in skin or lymphoblastoid cell lines (Fig. 4b,c, Additional file 4: Figure S7b, Additional file 1: Table S16). These data substantiate the practical utility of CoRSIVs, in that noninvasive measurements of DNA methylation (for example, in peripheral blood) can provide an indication of epigenetic regulation in other tissues. To explore this further, we analyzed a recently published database encompassing 1319 "epigenome-wide association studies" (EWASdb) [40] (based on DNA methylation, many in peripheral blood). As expected (based on the systemic nature of CoRSIVs), this analysis showed that disease-associated CpG sites are $37 \%$ enriched in CoRSIVs relative to control regions $\left(\chi^{2}\right.$ test $\left.P=2.2 \times 10^{-4}\right)$ and $53 \%$ enriched in CoRSIVs relative to tDMRs $\left(\chi^{2}\right.$ test $P=6.5 \times 10^{-8}$ ) (Additional file 4: Figure S12e). To explore these associations in greater depth, we began by dividing the disease outcomes into cancer and non-cancer diseases, since the HM450 array is enriched for CpG sites known to be hypermethylated in cancer, and many epigenetic studies of cancer are based on comparing tumor vs. normal tissue, which is not relevant to the systemic nature of CoRSIVs. Whereas CoRSIV probes were not enriched for disease associations with cancer (Additional file 4: Figure S14), they did show strong associations to noncancer diseases (Additional file 4: Figure S15). The greatest enrichments were found for "deletion or duplication of chromosome 7q11.23," "immunodeficiency, centromeric instability and facial abnormalities (ICF) syndrome," 



Fig. 4 CoRSIVs show influence of periconceptional environment, correlate with gene expression, and are associated with human disease. a Seasonal variation of blood methylation at CoRSIVs, controls, and tDMRs in 233 Gambian 2-year olds [13]. Data represent CpGs showing significant seasonal variation (FDR < 20\%, see "Methods"). Predicted methylation maxima for CpGs within CoRSIVs occur in conceptions at the peak of the Gambian rainy season (July-September); minima fall within the Gambian dry season (Jan-April). Seasonal patterns at control regions and tDMRs are less pronounced (Fisher's exact test $P>0.8$ ). $\mathbf{b}$ Results of analysis linking gene expression in adipose tissue, skin, and lymphoblastoid cell lines (LCL) vs. methylation in adipose tissue [26] for 645 gene-associated CoRSIVs that are informative on the HM450 array. Venn diagrams show that for most genes that show a significant association (Spearman $P<0.05$ ) between methylation and expression in adipose tissue, methylation in adipose tissue is also associated with expression in skin or LCL. c Examples of expression vs. CoRSIV methylation data in all three tissues at the MR/1 promoter (left), the CNDP2 gene body (middle), and the $3^{\prime}$ end of SSNA1 (right). d Summary of an automated PubMed literature-search using PubTator. Shown are MESH code labels corresponding to the top 100 human diseases linked to CoRSIV-associated genes

"asthma," "down syndrome," and "human immunodeficiency virus." Notably, though several of these relate to immune function, none of the probes driving these enrichments were within the CoRSIV-rich MHC region on chromosome 6.

Although CoRSIVs are depleted in and around genes (Fig. 2f), there are thousands of CoRSIV-associated genes. As another way to explore potential phenotypic consequences of epigenetic variation at CoRSIVs, we used GREAT [41] to perform gene ontology analysis (Additional file 4: Figure S16). The main significant enrichments (vs. genomic background) relate to the high density of CoRSIVs identified in the MHC locus. Otherwise, CoRSIVs were not associated with specific cellular processes or components. We used an automated literature search tool (PubTator) [42] to search PubMed and identify diseases linked to the 3127 CoRSIV-associated genes. This analysis initially found that, compared to all genes listed in PubMed, CoRSIV-associated genes are enriched for associations with hundreds of diseases, particularly those involving the brain (Additional file 1: Tables S17, S18, Additional file 4: Figure S12c). Recent studies have shown that genes expressed in the brain (particularly in neurons) tend to be longer than average [43], so we tested whether our analysis based on gene-body overlap could bias toward longer genes. Indeed, relative to all PubTator genes, CoRSIV-associated genes are enriched for long genes (Additional file 4: Figure S12d). After adjusting for 
gene length, CoRSIV-associated genes were no longer associated with diseases involving the brain (Additional file 1: Table S17). Nonetheless, thousands of CoRSIV-associated genes are implicated in a wide range of human diseases including neoplasms, mental disorders, digestive, nervous system, and cardiovascular diseases (Fig. 4d, Additional file 1: Table S18). Recent reports highlight several examples, including SPATC1L in male infertility [44], PM20D1 in Alzheimer's disease [29], and DUSP22 in lymphoma [45].

\section{Discussion}

Here, we have uncovered, charted, and characterized a previously unrecognized level of molecular individuality in humans. Although CoRSIVs were identified in Caucasians, we validated systemic interindividual epigenetic variation at these loci in an Asian cohort and confirmed interindividual variation and documented an influence of periconceptional environment in rural Africans. Hence, together our data indicate that CoRSIVs are an ancestral and universal feature of the human genome.

Our study is not without limitations. Since our screen included only 10 individuals, all Caucasian, this first CoRSIV atlas is incomplete. For example, we did not detect a known CoRSIV at VTRNA2-1 $[6,46]$ because all 10 individuals happen to exhibit normal $(\sim 50 \%)$ methylation at the locus. Another potential caveat (which extends to most studies using GTEx samples) is that many of the donors studied were at late stages of disease (Additional file 1: Table S5). Future screens surveying additional individuals (and diverse ethnic groups) will undoubtedly identify additional human CoRSIVs. Lastly, our use of the term "systemic" merits clarification. Our intended meaning is that systemic epigenetic variants are generally consistent across all tissues and cell types. Indeed, although our screen was based on thyroid, heart, and brain, our various validation studies additionally confirmed interindividual variation in liver, kidney, peripheral blood, and fingernail. Nonetheless, given the $\sim 200$ cell types in the human body, it is impossible to rule out cell-type specific effects at some of these loci. For example, it was recently shown that methylation at the VTRNA2-1 CoRSIV lacks interindividual variation specifically in the cerebellum (but not in the cerebral cortex) [47].

Our analyses indicate that, at some of these loci, systemic interindividual epigenetic variation may not be determined by local genetic control. This somewhat surprising finding is consistent with the most powerful whole-genome analysis of genetic effects on methylation [14], which concluded that most interindividual variation in DNA methylation in human peripheral blood and adipose tissue is not associated with genetic variation. Future targeted analyses-sufficiently powered to detect both cis and trans effects-will be required to determine if a subset of CoRSIVs are, indeed, metastable epialleles. Identifying a substantial number of metastable epialleles in the human genome-including some under partial genetic influence $[5,13]$-would provide support for the thesis that stochastic interindividual epigenetic variation is evolutionarily advantageous and "hard wired" into the human genome [48]. Given the ability to now use existing banked DNA samples (such as from peripheral blood) to broadly assay systemic and stable individual epigenetic variants, we anticipate that this CoRSIV atlas will accelerate future progress in the field of epigenetics and human disease [49].

\section{Methods}

Study populations

Tissue samples from the NIH Genotype-Tissue Expression (GTEx) program were collected during rapid autopsy or organ transplant settings, so most organs will be free of major disease processes [9]. Inclusion criteria were as follows: donor age 50-69, no morbid obesity (BMI 18.5-35), postmortem interval (death to tissue collection) $<24 \mathrm{~h}$, no recent blood transfusions, no metastatic cancer, and no chemotherapy or radiation therapy in the last 2 years. The 10 Caucasian donors were balanced by sex and were selected based on the availability of all three tissues (thyroid, heart, brain).

\section{Analytical approach}

Deep whole-genome bisulfite-sequencing (WGBS) was performed on 30 tissue samples and were preprocessed as described in Additional file 3: Supplementary methods. To maximize genomic coverage, we applied a two-stage approach to identify regions of SIV. First, all reads from each individual were combined to calculate individuallevel average methylation for each bin, and individually correlated regions of methylation (bin-bin $R \geq 0.71$ $\left.\left(R^{2} \geq 0.5\right)\right)$ were built in a step-wise fashion $[11,12]$ (for details see Additional file 3: Supplementary Methods). Then, for each such region, inter-tissue correlation (ITC) of average methylation was assessed across all tissue-type pairs. Regions yielding a minimum ITC $\geq 0.71$ were identified as "correlated regions of SIV" (CoRSIVs). Five complementary approaches were considered to evaluate the influence of genotype on CoRSIVs. The associations of CoRSIVs with many genomic features such as genes, sub-telomeric regions, repetitive elements, CpG islands, transcription factor binding sites, and periconceptional environment were evaluated. An assessment of CoRSIV-associated human gene and disease associations were conducted based on PubMed using Pubtator framework (see Additional file 3: Supplementary methods, for details). 


\section{Statistical analysis}

A permutation test was developed to evaluate the probability of CoRSIVs arising by chance. In each of 100,000 permutations, we scrambled subject IDs within each tissue type, computed ITCs for each of 1000 randomly selected blocks, and counted how many times a minimum ITC $\geq 0.71$ was obtained (see Additional file 3: Supplementary methods, for details). Statistical significance of enrichments/depletions of CoRSIVs in various genomic contexts was calculated relative to two reference sets (controls, tDMRs) using $\chi^{2}$ tests. Associations of various epigenome states with CoRSIVs were analyzed by Fisher's exact test. Analyses of mQTL using the genotype data from 10 GTEx donors used linear regression; CoRSIVs with regression coefficient $\beta \geq 10, R^{2} \geq 0.5$, and FDR $<5 \%$ were considered positive for mQTL. Associations between CoRSIV methylation and gene expression were evaluated based on the Spearman correlation, adjusted for multiple testing using the Benjamini-Hochberg method. Enrichment of seasonal effects at CoRSIVs and the two reference sets was determined using Fourier regression models and Fisher's exact test.

\section{Additional files}

Additional file 1: Supplementary Tables S1, S3-S19. (XLS 26942 kb)

Additional file 2: Supplementary Table S2. (CSV 31408 kb)

Additional file 3: Supplementary methods. (DOCX $359 \mathrm{~kb}$ )

Additional file 4: Supplementary Figures. (DOCX 15207 kb)

\section{Acknowledgements}

We thank Yiying Gao (Beijing Genomics Institute) for facilitating the sequencing, and Adam Gillum (Baylor College of Medicine) for assistance with the figures.

\section{Funding}

Generation of the WGBS data was conducted by the Beijing Genomics Institute with funding from the Bill \& Melinda Gates Foundation. Computational analysis was supported by NIH/NIDDK (grant number 1R01DK111522), the Cancer Prevention and Research Institute of Texas (grant number RP170295), and USDA/ARS (CRIS 3092-5-001-059). The ENID Trial was jointly funded by the UK Medical Research Council (MRC) and the Department for International Development (DFID) under the MRC/DFID Concordat agreement (MRC Program MC-A760-5QX00). The methylation analysis of Gambian samples was supported by the Bill \& Melinda Gates Foundation (grant no: OPP1 066947), and we acknowledge the work of Z. Herceg, M. N. Routledge, Y. Y. Gong, and $\mathrm{H}$. Hernandez-Vargas in acquiring these data. Next-generation sequencing library preparation was conducted at the Baylor College of Medicine Functional Genomics Core, which is partially supported by the NIH shared instrument grant number S100D023469 to RC. The infant twin studies were supported by $\mathrm{NIH} / \mathrm{NICHD}$ (grant number 1R21HD087860). GH is supported by a Sir Henry Dale Fellowship jointly funded by the Wellcome Trust and the Royal Society (Grant Number 098386/Z/12/Z).

\section{Availability of data and materials}

The raw sequencing data from the 30 methylomes have been deposited in dbGAP (accession \# phs001746.v1.p1) [50].

\section{Authors' contributions}

RAW, CC, and AMP obtained funding. CG, CAS, CC, MJS, NJK, HM, and JDD performed data analysis. $\mathrm{CC}, \mathrm{GH}$, and RAW advised and guided the data analysis. CG, CC, GH, MJS, AMP, and RAW contributed to the interpretation of results. SEM and AMP set up and led the ENID study. EL and MSB performed pyrosequencing validation studies. $\mathrm{YL}$ and $\mathrm{RC}$ were responsible for bisulfite library construction and quality control. $A C W, K R H, M G$, and $A B H$ set up and provided samples from the neonatal twin study. RAW and CC conceived the initial CoRSIV study design and analysis strategy. CG and RAW wrote the manuscript, with input from all coauthors. All authors read and approved the final manuscript;

Ethics approval and consent to participate

Not applicable.

\section{Consent for publication}

Not applicable.

\section{Competing interests}

The authors declare that they have no competing interests.

\section{Publisher's Note}

Springer Nature remains neutral with regard to jurisdictional claims in published maps and institutional affiliations.

\section{Author details}

${ }^{1}$ USDA/ARS Children's Nutrition Research Center, Department of Pediatrics, Baylor College of Medicine, Houston, TX, USA. ²Department of Genetics, Evolution and Environment, UCL Genetics Institute, University College London, London WC1E 6BT, UK. ${ }^{3}$ MRC Unit The Gambia at London School of Hygiene and Tropical Medicine, Keneba, The Gambia. ${ }^{4}$ Department of Women and Children's Health, King's College London, London, UK.

${ }^{5}$ Department of Obstetrics and Gynecology, Baylor College of Medicine, Houston, TX, USA. ${ }^{6}$ Department of Pediatrics - Neonatology, Baylor College of Medicine, Houston, TX, USA. 'Human Genome Sequencing Center, Baylor College of Medicine, Houston, TX, USA. ${ }^{8}$ Department of Molecular and Human Genetics, Baylor College of Medicine, Houston, TX, USA. 'Department of Molecular and Cellular Biology, Baylor College of Medicine, Houston, TX, USA.

Received: 26 April 2019 Accepted: 6 May 2019

Published online: 03 June 2019

\section{References}

1. Dor $Y$, Cedar H. Principles of DNA methylation and their implications for biology and medicine. Lancet. 2018;392:777-86.

2. Smith ZD, Meissner A. DNA methylation: roles in mammalian development. Nat Rev Genet. 2013;14:204-20.

3. Schultz MD, He Y, Whitaker JW, Hariharan M, Mukamel EA, Leung D, Rajagopal N, Nery JR, Urich MA, Chen $H$, et al. Human body epigenome maps reveal noncanonical DNA methylation variation. Nature. 2015;523:212-6.

4. Ziller MJ, Gu H, Muller F, Donaghey J, Tsai LT, Kohlbacher O, De Jager PL, Rosen ED, Bennett DA, Bernstein BE, et al. Charting a dynamic DNA methylation landscape of the human genome. Nature. 2013;500:477-81.

5. Kessler NJ, Waterland RA, Prentice AM, Silver MJ. Establishment of environmentally sensitive DNA methylation states in the very early human embryo. Sci Adv. 2018;4:eaat2624.

6. Silver MJ, Kessler NJ, Hennig BJ, Dominguez-Salas P, Laritsky E, Baker MS, Coarfa C, Hernandez-Vargas H, Castelino JM, Routledge MN, et al. Independent genomewide screens identify the tumor suppressor VTRNA2-1 as a human epiallele responsive to periconceptional environment. Genome Biol. 2015;16:118.

7. Waterland RA, Jirtle RL. Transposable elements: targets for early nutritional effects on epigenetic gene regulation. Mol Cell Biol. 2003;23:5293-300.

8. Waterland RA, Kellermayer R, Laritsky E, Rayco-Solon P, Harris RA, Travisano M, Zhang W, Torskaya MS, Zhang J, Shen L, et al. Season of conception in rural Gambia affects DNA methylation at putative human metastable epialleles. PLoS Genet. 2010;6:e1001252.

9. Consortium G, Laboratory DA. Coordinating Center -Analysis Working G, Statistical Methods groups-Analysis Working G, Enhancing Gg, Fund NIHC, Nih/Nci, Nih/Nhgri, Nih/Nimh, Nih/Nida, et al: genetic effects on gene expression across human tissues. Nature. 2017;550:204-13.

10. Bock C. Analysing and interpreting DNA methylation data. Nat Rev Genet. 2012;13:705-19. 
11. Schmitz RJ, Schultz MD, Urich MA, Nery JR, Pelizzola M, Libiger O, Alix A, McCosh RB, Chen H, Schork NJ, Ecker JR. Patterns of population epigenomic diversity. Nature. 2013;495:193-8.

12. Liu Y, Li X, Aryee MJ, Ekstrom TJ, Padyukov L, Klareskog L, Vandiver A, Moore AZ, Tanaka T, Ferrucci L, et al. GeMes, clusters of DNA methylation under genetic control, can inform genetic and epigenetic analysis of disease. Am J Hum Genet. 2014;94:485-95.

13. Van Baak TE, Coarfa C, Dugue PA, Fiorito G, Laritsky E, Baker MS, Kessler NJ, Dong J, Duryea JD, Silver MJ, et al. Epigenetic supersimilarity of monozygotic twin pairs. Genome Biol. 2018;19:2.

14. Busche S, Shao X, Caron M, Kwan T, Allum F, Cheung WA, Ge B, Westfall S, Simon MM, Multiple tissue human expression $\mathrm{R}$, et al. Population whole-genome bisulfite sequencing across two tissues highlights the environment as the principal source of human methylome variation. Genome Biol. 2015;16:290.

15. Farlik M, Halbritter F, Muller F, Choudry FA, Ebert P, Klughammer J, Farrow S, Santoro A, Ciaurro V, Mathur A, et al. DNA methylation dynamics of human hematopoietic stem cell differentiation. Cell Stem Cell. 2016;19:808-22.

16. Hannon E, Lunnon K, Schalkwyk L, Mill J. Interindividual methylomic variation across blood, cortex, and cerebellum: implications for epigenetic studies of neurological and neuropsychiatric phenotypes. Epigenetics. 2015;10:1024-32.

17. Song MA, Brasky TM, Marian C, Weng DY, Taslim C, Llanos AA, Dumitrescu RG, Liu Z, Mason JB, Spear SL, et al. Genetic variation in one-carbon metabolism in relation to genome-wide DNA methylation in breast tissue from heathy women. Carcinogenesis. 2016;37:471-480.

18. Fortin JP, Hansen KD. Reconstructing A/B compartments as revealed by $\mathrm{Hi}-\mathrm{C}$ using long-range correlations in epigenetic data. Genome Biol. 2015;16:180.

19. Wall JD, Pritchard JK. Haplotype blocks and linkage disequilibrium in the human genome. Nat Rev Genet. 2003:4:587-97.

20. Smith EM, Lajoie BR, Jain G, Dekker J. Invariant TAD boundaries constrain cell-type-specific looping interactions between promoters and distal elements around the CFTR locus. Am J Hum Genet. 2016;98:185-201.

21. Rakyan VK, Hildmann T, Novik KL, Lewin J, Tost J, Cox AV, Andrews TD, Howe KL, Otto T, Olek A, et al. DNA methylation profiling of the human major histocompatibility complex: a pilot study for the human epigenome project. PLoS Biol. 2004;2:e405.

22. Consortium EP. An integrated encyclopedia of DNA elements in the human genome. Nature. 2012;489:57-74.

23. Roadmap Epigenomics C, Kundaje A, Meuleman W, Ernst J, Bilenky M, Yen A, Heravi-Moussavi A, Kheradpour P, Zhang Z, Wang J, et al. Integrative analysis of 111 reference human epigenomes. Nature. 2015;518:317-30.

24. Bernstein BE, Mikkelsen TS, Xie X, Kamal M, Huebert DJ, Cuff J, Fry B, Meissner A, Wernig M, Plath $\mathrm{K}$, et al. A bivalent chromatin structure marks key developmental genes in embryonic stem cells. Cell. 2006;125:315-26.

25. Cooper GM, Stone EA, Asimenos G, Program NCS, Green ED, Batzoglou S, Sidow A. Distribution and intensity of constraint in mammalian genomic sequence. Genome Res. 2005;15:901-13.

26. Grundberg E, Meduri E, Sandling JK, Hedman AK, Keildson S, Buil A, Busche S, Yuan W, Nisbet J, Sekowska M, et al. Global analysis of DNA methylation variation in adipose tissue from twins reveals links to disease-associated variants in distal regulatory elements. Am J Hum Genet. 2013;93:876-90.

27. Shi J, Marconett CN, Duan J, Hyland PL, Li P, Wang Z, Wheeler W, Zhou B, Campan M, Lee DS, et al. Characterizing the genetic basis of methylome diversity in histologically normal human lung tissue. Nat Commun. 2014;5:3365.

28. Do C, Shearer A, Suzuki M, Terry MB, Gelernter J, Greally JM, Tycko B. Genetic-epigenetic interactions in cis: a major focus in the post-GWAS era. Genome Biol. 2017;18:120.

29. Sanchez-Mut JV, Heyn H, Silva BA, Dixsaut L, Garcia-Esparcia P, Vidal E, Sayols S, Glauser L, Monteagudo-Sanchez A, Perez-Tur J, et al. PM20D1 is a quantitative trait locus associated with Alzheimer's disease. Nat Med. 2018; 24:598-603.

30. Consortium G. The genotype-tissue expression (GTEx) project. Nat Genet. 2013:45:580-5.

31. Bonder MJ, Luijk R, Zhernakova DV, Moed M, Deelen P, Vermaat $M$, van Iterson M, van Dijk F, van Galen M, Bot J, et al. Disease variants alter transcription factor levels and methylation of their binding sites. Nat Genet. 2017:49:131-8

32. Gaunt TR, Shihab HA, Hemani G, Min JL, Woodward G, Lyttleton O, Zheng J, Duggirala A, McArdle WL, Ho K. Systematic identification of genetic influences on methylation across the human life course. Genome Biol. 2016;17:61.

33. Ng B, White CC, Klein H-U, Sieberts SK, McCabe C, Patrick E, Xu J, Yu L, Gaiteri C, Bennett DA. An XQTL map integrates the genetic architecture of the human brain's transcriptome and epigenome. Nat Neurosci. 2017;20:1418

34. Firth HV, Richards SM, Bevan AP, Clayton S, Corpas M, Rajan D, Van Vooren S, Moreau Y, Pettett RM, Carter NP. DECIPHER: database of chromosomal imbalance and phenotype in humans using Ensembl resources. Am J Hum Genet. 2009;84:524-33

35. Genomes Project C, Auton A, Brooks LD, Durbin RM, Garrison EP, Kang HM, Korbel JO, Marchini JL, McCarthy S, McVean GA, Abecasis GR. A global reference for human genetic variation. Nature. 2015;526:68-74.

36. Rakyan VK. Metastable epialleles in mammals. Trends Genet. 2002;18:348-51.

37. Dominguez-Salas P, Moore SE, Baker MS, Bergen AW, Cox SE, Dyer RA, Fulford AJ, Guan Y, Laritsky E, Silver MJ, et al. Maternal nutrition at conception modulates DNA methylation of human metastable epialleles. Nat Commun. 2014;5:3746

38. Waterland RA. Maternal methyl supplements increase offspring DNA methylation at Axin fused. Genesis (New York, NY : 2000). 2006:44:401-6.

39. Waterland RA, Michels KB. Epigenetic epidemiology of the developmental origins hypothesis. Annu Rev Nutr. 2007;27:363-88.

40. Liu D, Zhao L, Wang Z, Zhou X, Fan X, Li Y, Xu J, Hu S, Niu M, Song X, et al. EWASdb: epigenome-wide association study database. Nucleic Acids Res. 2018.

41. McLean CY, Bristor D, Hiller M, Clarke SL, Schaar BT, Lowe CB, Wenger AM, Bejerano G. GREAT improves functional interpretation of cis-regulatory regions. Nat Biotechnol. 2010;28:495-501.

42. Wei CH, Kao HY, Lu Z. PubTator: a web-based text mining tool for assisting biocuration. Nucleic Acids Res. 2013;41:W518-22.

43. Gabel HW, Kinde B, Stroud H, Gilbert CS, Harmin DA, Kastan NR, Hemberg M, Ebert DH, Greenberg ME. Disruption of DNA-methylation-dependent long gene repression in Rett syndrome. Nature. 2015;522:89.

44. Kim J, Kwon JT, Jeong J, Kim J, Hong SH, Kim J, Park ZY, Chung KH, Eddy EM, Cho C. SPATC1L maintains the integrity of the sperm head-tail junction. EMBO Rep. 2018;e45991:1-19.

45. Hapgood G, Savage KJ. The biology and management of systemic anaplastic large cell lymphoma. Blood. 2015;126:17-25.

46. Carpenter BL, Zhou W, Madaj Z, DeWitt AK, Ross JP, Gronbaek K, Liang G, Clark SJ, Molloy PL, Jones PA. Mother-child transmission of epigenetic information by tunable polymorphic imprinting. Proc Natl Acad Sci U S A. 2018;115:E11970-7.

47. Marzi SJ, Meaburn EL, Dempster EL, Lunnon K, Paya-Cano JL, Smith RG, Volta M, Troakes C, Schalkwyk LC, Mill J. Tissue-specific patterns of allelicallyskewed DNA methylation. Epigenetics. 2016;11:24-35.

48. Feinberg AP, Irizarry RA. Evolution in health and medicine Sackler colloquium: stochastic epigenetic variation as a driving force of development, evolutionary adaptation, and disease. Proc Natl Acad Sci U S A. 2010;107(Suppl 1):1757-64.

49. Waterland RA, Garza C. Potential mechanisms of metabolic imprinting that lead to chronic disease. AmJClinNutr. 1999;69:179-97.

50. Gunasekara CS, CA; Laritsky, E; Baker, MS; MacKay, H; et. al.: A genomic atlas of systemic interindividual epigenetic variation in humans. dbGAP. 2019 https://www.ncbi.nlm.nih.gov/projects/gap/cgi-bin/study.cgi?study_id= phs001746.v1.p1. Accessed 6 May 2019.

\section{Ready to submit your research? Choose BMC and benefit from}

- fast, convenient online submission

- thorough peer review by experienced researchers in your field

- rapid publication on acceptance

- support for research data, including large and complex data types

- gold Open Access which fosters wider collaboration and increased citations

- maximum visibility for your research: over $100 \mathrm{M}$ website views per year

At $\mathrm{BMC}$, research is always in progress.

Learn more biomedcentral.com/submissions 